\title{
Interactive comment on "The downscaling and adjustment method ADAMONT v1.0 for climate projections in mountainous regions applicable to energy balance land surface models" by $\mathrm{D}$. Verfaillie et al.
}

D. Verfaillie et al.

deborah.verfaillie@meteo.fr

Received and published: 27 January 2017

Please find our response in the attached pdf file.

Please also note the supplement to this comment:

http://www.geosci-model-dev-discuss.net/gmd-2016-168/gmd-2016-168-AC3-

supplement.pdf 